\title{
Mozart et la tragédie française
}

\section{Charles Mazouer}

\section{OpenEdition}

\section{Journals}

Édition électronique

URL : http://journals.openedition.org/studifrancesi/8158

DOI : 10.4000/studifrancesi.8158

ISSN : 2421-5856

\section{Éditeur}

Rosenberg \& Sellier

\section{Édition imprimée}

Date de publication : 1 mai 2009

Pagination : 112-119

ISSN : 0039-2944

\section{Référence électronique}

Charles Mazouer, « Mozart et la tragédie française », Studi Francesi [En ligne], 157 (LIII | I) | 2009, mis en ligne le 30 novembre 2015, consulté le 09 janvier 2021. URL : http://journals.openedition.org/ studifrancesi/8158; DOI : https://doi.org/10.4000/studifrancesi.8158

\section{(c) (i) (2) $\Theta$}

Studi Francesi è distribuita con Licenza Creative Commons Attribuzione - Non commerciale - Non opere derivate 4.0 Internazionale. 


\section{Mozart et la tragédie française}

Par trois fois, à travers les livrets qui lui furent donnés pour composer la musique, Mozart rencontra la tragédie française: avec Mitridate, rè di Ponto (K 87) en 1770, avec Idomeneo, rè di Creta (K 366) en 1781, et avec Thamos, König in Aegypten (K 345); nous éliminerons cette dernière œuvre: il s'agit de quelques numéros d'une musique de scène primitivement écrite pour un drame allemand de von Gebler, en 1773, qui fut remaniée et réutilisée en 1779 pour l'adaptation allemande, sous le titre de Lanassa, d'une tragédie du Français Lemierre: La Veuve de Malabar. Restent donc deux opéras, participant de la même forme, dotée de ses formules obligées, de l'opera seria, pour lesquels Mozart fut très largement tributaire de ses librettistes; non sans jugement ni sans changements, l'un - Vittorio Cigna-Santi - avait suivi de près le célèbre Mithridate de Racine (1673), et l'autre - l'abbé Varesco - avait fait de même avec le livret composé et publié pour la première fois en 1712 par Danchet pour l'Idoménée mis en musique par Campra. Racine d'abord, de manière éclatante; Danchet ensuite, mais trop admirateur de Racine pour qu'on ne retrouve pas chez lui, à la faveur du sujet légendaire, de vives réminiscences raciniennes². Quoi qu'il en soit, de Racine ou de Danchet à Mozart le musicien, par le relais des modestes mais bons librettistes, la distance esthétique est grande.

Pour l'apprécier, je partirai des catégories traditionnelles: la transcendance tragique $^{3}$ et son piège, les passions et les dénouements. Se déterminera ainsi la position de Mozart vis-à-vis de la tragédie française ${ }^{4}$.

$$
* * *
$$

(1) Repris aux pages 121-182 du Théâtre de M. Danchet, t. III, Paris, 1751.

(2) Bien relevées par Jean-Michel BRÈQuE, «Les vertus paradoxales d'un livret imparfait», L'AvantScène Opéra, n. 89, 1996, pp. 16-21.

(3) Voir l'étude classique d'Henri GouHier, Le Théâtre et l'existence, nouvelle édition, Paris, Vrin, 1973.

(4) Etudes utilisées: Gustave Lanson, Esquisse d'une histoire de la tragédie française (1920), nouvelle édition, Paris, Champion, 1954; René GuIET, L'Evolution d'un genre: le livret d'opéra en France de Gluck à la Révolution (1774-1793), Northampton (Mass.), Smith College, 1937; Karl BARTH, Wolfgang Amadeus Mozart; 1756-1956 (1956), Zürich, Theologischer Verlag, 1978; Jean-Victor HocQuARD, La Pensée de Mozart, Paris, Seuil, 1958; Cuthbert GIRDLESTONE, Tragédie et tragédie en musique (16731727), «C.A.I.E.F.», n. 17, 1965, pp. 9-23; Jean et Brigitte Massin, Wolfgang Amadeus Mozart, Paris, Fayard, 1970; Cuthbert Girdlestone, La Tragédie en musique (1673-1750) considérée comme genre littéraire, Genève, Droz, 1972, et Idoménée...Ido- meneo. Transformations d'un thème (1699-1781), Recherches sur la musique française classique, XIII, 1973, Paris, A. et J. PICARD, pp. 102-132; Remy STRICKER, Mozart et ses opéras. Fiction et vérité, Paris, Gallimard, 1980 (repris dans «Tel», 1987); Jean-Victor Hocquard, Idoménée, Paris, AubierMontaigne, 1982 (Les grands opéras de Mozart); Mozart, "Mithridate", «L'Avant-Scène Opéra», n. 54, 1983; Charles Mazouer, "Idomeneo, rè di Creta": Mozart et la tragédie, «Revue belge de musicologie», vol. XXXVI-XXXVIII, 1982-1984, pp. 133-144; Jean-Victor HocQuARD, La Clémence de Titus et opéras de jeunesse, Paris, Aubier, 1986 (Les grands opéras de Mozart); Mozart, "Idoménée", «L'Avant-Scène Opéra», n. 89, 1986; Jean-Victor Hocquard, Mozart. L'amour. La mort, Paris, Editions Garamond-Archimbaud, 1987 (Paris, Lattès, 1992); Dictionnaire Mozart, dir. H. C. RobBIns Landon, Paris, Lattès, 1990 (Paris, Fayard, 1997); Annie Paradis, Mozart, l'opéra enchanté, Paris, Fayard, 1999; Pierre Michot, Mozart, opéras. Mode d'emploi, Paris, L'Avant-Scène Opéra, 2006. 
On définit communément le tragique comme la présence d'une transcendance qui s'acharne à détruire les mortels, libres assurément et donc ou coupables ou innocents. Cet univers tragique est infiniment plus présent dans Idomeneo que dans Mitridate.

Le livret de Danchet (que le librettiste y croie ou non est une autre question) maintient la présence d'un destin ennemi des mortels. Vénus, Neptune surtout, qui apaise la tempête mais rappelle à Idoménée son vœu de sacrifier le premier homme rencontré (II, 2) - ce sera son fils - appesantissent leur vengeance sur les hommes; le roi de Crète ne pourra pas échapper à son vœu ni épargner son fils: Neptune sort des enfers et excite les Furies qui rendent fou Idoménée et le poussent à tuer lui-même son fils Idamante. Les héros humains sont broyés par la loi d'airain de la vengeance de Neptune.

Varesco, très largement fidèle à Danchet, le retouche souvent avec talent, seul ou guidé par l'instinct dramatique de Mozart. Et, à partir de ce livret, le musicien écrivit une musique profondément tragiques. Dès l'ouverture, grandiose portique en ré majeur, l'atmosphère tragique est posée et rendu sensible le destin qui pèsera sur tout l'opéra. Et trois ou quatre chœurs remarquablement répartis dans chacun des trois actes suffisent à manifester la présence écrasante, effrayante du dieu Neptune, dont la volonté réduit à rien celle des Crétois et de leurs princes. Car c'est Neptune, le dieu de la mer lui seul, qui impose son ordre - déchaînant et calmant les flots, apparaissant puis s'enfonçant dans la mer après avoir regardé «con occhio torvo $e$ minaccevole» Idomeneo qui vient de faire son vœu terrible, intervenant brutalement, à la fin de l'acte II, pour punir, puisque Idomeneo est coupable de n'avoir pas tenu sa promesse, voulant imposer enfin à son Grand Prêtre, que soutient le peuple, le sacrifice d'Idamante.

La machine infernale s'est mise en route quand Neptune, qui poursuit de sa vindicte le vainqueur des Troyens, a tendu un piège à Idomeneo, l'obligeant à un vœu égoïste et affreux pour se sauver du naufrage, lui extorquant littéralement une victime humaine («m'estorse in voto umana vittima»). Le dieu vindicatif a su contraindre Idomeneo à la culpabilité et poursuivra sans relâche le coupable, lié à sa promesse. On n'échappe pas à l'engrenage tragique. L'action de l'opéra accule Idomeneo au barbare sacrifice. Malgré sa révolte contre le dieu méchant, malgré ses tentatives pour échapper à sa promesse - tout espoir d'apaisement, toute possibilité d'issue pour échapper au cruel destin, toute promesse de bonheur possible ressortissant à l'illusion tragique, à l'ironie tragique qui ne met que mieux en valeur la marche inéluctable du malheur -, Idomeneo ne semble pouvoir reculer devant le sacrifice de son fils.

La dramaturgie, les effets scéniques - pensons à une mise en scène moderne comme celle de Jean-Pierre Ponnelle à Salzbourg -, les recitativi secchi ou accompagnati expriment ou rendent présent et oppressant le destin tragique. Mais les chants confiés par le musicien à Idomeneo, à la fois victime et coupable, ponctuent la marche de l'action tragique, formulant à leur manière le sentiment du destin. A l'instar du roi, tous les autres personnages, innocents quant à eux, sont entraînés et risquent d'être noyés par la vague tragique, Idamante et Ilia au premier chef, emportés dans des situations tragiques qui les dépassent, les déchirent et les conduiraient à la mort. Ilia, fille du vaincu Priam, captive du vainqueur chez les ennemis de son peuple, mais sauvée par le charmant Idamante qu'elle aime, retrouve un topos de la tragédie française classique, partagée qu'elle est entre la haine pour les ennemis et son amour pour un des ennemis, le fils de son bourreau. Un sort plus affreux attend Idamante,

(5) Voir CH. Mazouer, "Idomeneo, rè di Creta": Mozart et la tragédie, article cité, pp. 136-140, pour davantage de détails. 
froidement accueilli par son père mais finalement soumis au cruel destin de celui-ci quand il se sait promis au sacrifice. Comme Ilia, qui veut se substituer à lui au dernier moment, Idamante serait une hostie consentante, happée par l'exigence mortelle du dieu et du destin.

Sur un livret longtemps fidèle à Danchet, Mozart, avec une musique splendide et puissamment efficace, a amplifié et comme revivifié, rendue profondément tragique une atmosphère d'un tragique plutôt discret chez le librettiste français. Mais un tel tragique, avec cette histoire légendaire du cycle troyen, fait plutôt penser à la tragédie grecque qu'à notre tragédie classique.

L'adolescent de quatorze ans ne disposait sans doute pas des ressources humaines et esthétiques pour atteindre une telle puissance quand il composa, onze ans avant Idomeneo, la musique de Mitridate. D'autant que la tragédie de Racine, source du livret, n'était pas une fable mythologique propice à l'action des dieux, mais un sujet historique, et que, d'autre part, le librettiste, parfaitement fidèle au scénario de Racine (à quelques modifications et édulcorations près), simplifie, affadit et aplatit la tragédie française, dont les finesses et la substance même lui échappent. Que put faire Mozart?

Assez fréquemment, les personnages de l'opéra (Aspasia, Mitridate, Sifare) évoquent le destin malheureux et s'adressent aux dieux. Mais sans cohérence: tantôt Dieu ( $\mathrm{n}^{\circ} 1$ ), le Dieu bon, tantôt le Ciel cruel (III, 3), tantôt, avec une belle incohérence chez Aspasia (III, 4), un panthéon qui associe un dieu méchant mettant en branle la Némésis et des dieux favorables aux innocents. Théologie de pacotille à laquelle personne ne croit - et surtout pas le musicien, qui jamais, dans cette enfilade d'airs qui constituent l'opera seria, ne donne le sentiment d'une transcendance tragique ${ }^{6}$.

A la vérité, chez Racine même, le plus grec de nos dramaturges pourtant, la transcendance religieuse s'efface. Ni dieux ni destin dans son Mithridate; plus exactement c'est le roi de Pont lui-même, de retour alors qu'on le croyait mort, qui risque d'être le destin des personnages, soupçonnant et débusquant les trahisons politiques et sentimentales de ses fils et de Monime, et décidé à s'en venger par leur mort. «Craignons pour vous, pour moi pour la reine elle-même», dit Pharnace à son frère Xipharès (I, 5, v. 351) en parlant d'un père cruel, juge sévère, jaloux furieux, nourrissant des haines et des vengeances inexorables. Le mal est chez les hommes, dans l'histoire, et non plus venu des dieux.

Mozart rend-il sensible cela dans les airs de Mitridate et de ses victimes? Plutôt que l'aria 10 où Mitridate chante son furor contre Farnace («Quel ribelle e quell'ingrato»), en I, 13, il faut écouter l'aria 17, en II, 14, où, dépouillé de toute pitié («Già pietà mi spoglio»), le roi, offensé comme père et comme amant, affiche sa violence et affirme sa vengeance («Voglio vendetta») en une musique inquiétante par son attaque, ses $f p$, ses marches chromatiques, ses sauts d'octave ${ }^{7}$. Malheureusement, d'autres sentiments, chantés ailleurs, ne permettent pas à Mitridate de conserver cette stature de destin tragique pour les autres, même si l'intrigue lui donne droit de mort sur eux. Quant à ses victimes, Racine avait fortement montré comment le retour du père avait plongé les fils et Monime dans un profond sentiment de culpabilité devant leur juge revenu. Chez Mozart, ce sentiment tragique s'estompe passablement: Farnace finit par se reconnaître coupable de manière grandiloquente et provocante (aria 16), mais avait oublié ce sentiment dans l'exercice de la trahison; Sifare se sent beaucoup plus

(6) Il est remarquable que, parfois et même assez souvent, les récitatifs aient plus de justesse, de vérité et donc d'effet sur l'auditeur que les airs.

(7) Voir aussi l'aria 20, en III, 3, où, en ce que
J.-V. Hocquard appelle des «glapissements», le roi décide, avant d'aller combattre les Romains, de faire mourir Aspasia. 
amoureux que coupable d'aimer celle que son père se destinait et, comme il se dit partagé entre l'amour et le devoir d'obéissance au père (autre topos tragique) et décidé à choisir le devoir, en III, 6, il va à la mort avec le sentiment de son innocence (aria 22); quant à Aspasia, soumise aux volontés brutales successives de Mitridate et de Farnace, elle se dit tourmentée par un affreux dilemme - autre noble et bien banal cas tragique: mourir avec celui qu'elle aime ou vivre et épouser, malgré sa répugnance, Mitridate, pour satisfaire sa gloire (II, 8 et aria 14). Comme si tous ces personnages de Mitridate tentaient, d'une manière touchante et un peu plate, de se hausser sur le cothurne tragique. Pour l'essentiel, la tragédie racinienne se voit trahie par l'opéra.

Qu'en est-il, de manière générale, des passions et des sentiments dans le malheur voulu par les dieux ou venu des hommes? La tragédie implique violence des passions, cruautés et souffrances aptes à plaire et à toucher, selon la formule racinienne de la Préface de Bérénice. Le musicien et ses librettistes adoucirent singulièrement cette âpre atmosphère: bourreaux moins dangereux, victimes touchantes dont le malheur n'est peut-être pas inéluctable.

Arrêtons-nous d'abord aux pères, puisque les deux opéras traitent, chacun à sa manière, de la paternité et des rapports entre père et fils.

Racine avait créé un Mithridate très inquiétant, un tyran ambigu et cruel, particulièrement dangereux dans ses amours et dans sa jalousie, et donc mortellement menaçant pour ses fils rivaux; sans doute avoue-t-il pour Xipharès une «tendresse cachée» et dit-il sa «joie», et «extrême», quand on lui donne l'assurance que seul l'autre fils, Pharnace, est un traître - au début de la tragédie, avant de savoir que Xipharès est son rival auprès de Monime. Tout se passe comme si l'opéra, s'emparant de l'indication racinienne, la travaillait, l'exploitait au risque de trahir Racine. Le récitatif accompagné de $\mathrm{I}, 13$, qui suit de fort près la réplique de Racine, insiste un peu plus sur la tendresse du père pour le fils si chéri («Quel figlio si caro»). Plus tard, en II, 4, quand Mitridate est persuadé qu'Aspasia aime Farnace et ignore encore les amours entre Sifare et Aspasia, Mozart compose une aria très fine $\left(n^{\circ} 12\right)$ qui fait s'opposer et se succéder à plusieurs reprises un allegro de colère à l'égard d'Aspasia (sol majeur) et un adagio tendre (en si bémol) pour Sifare («Tu, che fedel mi sei») comme si - remarque subtilement Jean-Victor Hocquard - la confiance et la tendresse à l'égard de Sifare étaient précaires. Certes; mais le musicien aura mis de la complaisance à rendre sensible le côté tendre de Mitridate.

Idomeneo va beaucoup plus loin dans ce sens: le musicien et son librettiste refusent de suivre Danchet qui avait fait d'Idoménée le rival de son fils auprès de la jeune captive $^{10}$. Dès son entrée en scène, Idomeneo est un homme accablé (I, 9) et bientôt, quand la première personne rencontrée est son fils, un père déchiré (I, 10). Torturé par l'atrocité d'avoir à sacrifier son fils, il cherchera continuellement à échapper, à imaginer des solutions (souvent invraisemblables et toujours vaines) pour éviter le sacrifice inévitable. Idomeneo est un père affectueux, soucieux de la vie et du bonheur amoureux de son fils dont il a surpris la passion pour Ilia, prêt à se substituer à l'innocent pour être sacrifié, incapable d'accomplir le geste déjà demandé à Abraham et à Agamemnon. Dans cet opéra, les rapports du père et du fils, qui font le nœud de

(8) II, 3, v. 467. A la scène suivante, II, 4, «ma tendre amitié» (v. 598) vise encore le fils préféré.

(9) Ibid., vers 511 sq.
(10) Et un vieux galant jaloux et menaçant, comme en III, 2 de son Idoménée. 
l'action, sont sous le signe de la tendresse réciproque et souffrante, à l'opposé d'un conflit familial tragique.

Et comme les opéras donnent une place capitale aux belles histoires d'amour longtemps malheureuses et souffrantes, mais qu'on sait promises au bonheur!

A sa manière, Mozart reprend et amplifie le pathétique des amours menacées par le roi de Pont, déjà mis en œuvre par Racine. L'annonce du retour de Mitridate cru mort entraîne un bel air de souffrance d'Aspasia, en sol mineur $\left(n^{\circ} 4:\right.$ : Nel sen mi palpita dolente il core»), particulièrement douloureux quand le personnage craint pour son amour; du bon Mozart à travers la stylistique d'un air de souffrance. Fautil se séparer (II, 7)? C'est à Sifare d'exhaler sa douleur dans un adagio tendrement douloureux ( $\mathrm{n}^{\circ} 13$ : «Lungi da te, mio bene») qui, ponctué d'interventions poignantes du cor, parcourt mélodiquement, ressasse la douleur de Sifare. Décide-t-on de mourir ensemble (II, 15)? C'est le duetto 18 où, d'abord, une mélodie bouleversante est entamée par Sifare, et bientôt reprise par Aspasia. Et il faudrait ajouter la belle cavatina 21 - un andante d'une douceur désespérée que chante Aspasia au moment de boire le poison. En toutes ces circonstances, la musique, dans la droite ligne de la tragédie mais en renforçant l'émotion, met en valeur la souffrance des malheureux amants.

Idomeneo donne une place centrale à l'histoire d'un amour tressé, jusqu'au dénouement, sur l'histoire tragique du vœu imprudent. Les airs d'Ilia jalonnent sa progression, de sa difficile naissance à l'aria 1 (comment la jeune Troyenne ne serait-elle pas révoltée, déchirée à l'idée d'aimer invinciblement le propre fils du bourreau de son peuple?) au sacrifice sublime de l'acte III (en III, 10, elle apparaît pour se substituer à Idamante qu'on est sur le point de sacrifier); et il faudrait s'arrêter aux étapes principales: son rêve de bonheur en Crète, avec Idamante pour époux et Idomeneo pour père, à qui, ayant dépassé la douleur, elle semble vouer des sentiments affectueux (II, 2 aria 11 en si bémol, qui montre parfaitement et la crampe de la douleur passée et dépassée, et le calme balancement du bonheur rêvé); l'aveu fait aux vents (III, 1, aria 19: «Zeffiretti lusinghieri») de manière délicate et gracieuse, avec des traits plus douloureux; enfin l'aveu fait directement à Idamante (III, 2, avec le duetto 20 a, où l'inquiétude d'Idamante est tendrement dissipée par Ilia, pour aboutir à un allegro de pure joie). Un tel amour, coloré d'une lumière presque pastorale, ne peut être malheureux! Pour avoir une version vraiment tragique, racinienne même, exactement, de l'amour sans espoir, c'est le rôle d'Elettra qu'il faut considérer, l'irréconciliée, qui se maintiendra jusqu'au bout dans le registre de la passion tragique - vengeance, fureur, cruauté, souffrance insupportable débouchant dans la folie finale ${ }^{11}$ : tout l'inverse de la douce Ilia.

Il règne finalement dans ces opéras un certain climat de souffrance apaisée et surmontée; il est très rare que la violence, la vengeance, le ressentiment persistent. Tout ce qui constitue le climat des passions et des douleurs tragiques, des sentiments violents dans le mal fait comme dans le mal subi, semble devoir être contesté par quelque sursaut touchant des personnages qui aspirent à un au-delà de l'émotion propre au tragique. On pourrait évoquer Ilia, justement, qui dépasse la douleur et le ressentiment, espère la réconciliation; mais je choisirai plutôt un personnage de Mitridate, nullement imaginé par Racine: Ismene, reine des Parthes, amoureuse sans espoir de Farnace mais à lui promise par Mitridate. L'accueil glacial que lui réserve Farnace

(11) Voir: I, 6, aria 4 (passion, jalousie, violence); II, 4, aria 13 (en parfait contraste; mais la joie d'Elettra d'embarquer avec Idamante repose sur une illusion que le destin va se charger de dénoncer dès la scène suivante); III, 10, aria 29a (air de fureur, d'une musique désarticulée, où le personnage se compare significativement à Oreste et à Ajax: «D'Oreste, d'Aiace | Ho in seno $i$ tormenti»). 
en I, 11 la plonge dans la souffrance, dans le tourment («tormento»), ce qui rend particulièrement poignante une méditation d'une vingtaine de mesures en sol mineur dans l'aria 9. En profite-t-elle pour obtenir vengeance? Nullement. Elle trouve déjà le moyen d'oublier cette souffrance pour apaiser, consoler Mitridate qui vient d'apprendre la trahison de Farnace (II, 12, aria 15). Mieux encore: en III, 1, elle refuse que son tourment se change en fureur (furor), que Farnace soit châtié, elle calme sa peine dans le gracieux balancement d'un allegro en sol majeur (aria 19) et formule l'espoir de regagner le cœur de l'ingrat. Bons sentiments, maîtrise de soi et espoirs touchants annoncent ce que pourront être le dénouement et l'adieu au tragique.

Le dénouement des opéras, en effet, scelle la réconciliation, le pardon et la fin des violences, l'effacement de la fatalité et la possibilité du bonheur - en particulier des amours heureuses. Certes, l'opera seria exigeait une telle fin; mais cette convention est librement et pleinement assumée par Mozart, qui par là s'éloigne peu ou prou de ses modèles de la tragédie française.

On sait que chez Racine, Mithridate mourant sauve Monime du poison et la donne à Xipharès qui vient d'arracher son père aux bataillons romains: «Vous seule me restez. Souffrez que je vous donne, | Madame ${ }^{12} . . . » ;$ il rend l'âme ensuite dans les bras de ce fils bien digne de lui, le frère Pharnace étant passé définitivement du côté des Romains. Dénouement heureux, vraiment? On peut en discuter. Mourant et sûr d'être imité après sa mort par ce fils qui partage sa résistance et sa haine contre les Romains, Mithridate ne fait pas, en tout cas, un grand sacrifice en laissant Monime à Xipharès, le fils pour lequel il a toujours nourri une tendresse cachée.

C'est à une réconciliation générale et authentique qu'on assiste dans l'opéra de Mozart. Pour commencer, nous avons droit à la véritable conversion de Farnace (III, 9). Tout à coup, la nature, le remords s'emparent de lui et déchirent le voile qui recouvrait sa raison; «Gia dagli occhi il velo è tolto», chante l'aria 24, dont l'andante en mi bémol s'élève paisiblement à la joie, et, dans l'allegretto suivant, à la décision d'emprunter désormais le chemin de la gloire et de l'honneur. Puis c'est à Mitridate de se convertir (III, 11), de reconnaître sa faute à l'égard d'Aspasia et de se faire pardonner le souvenir cruel de sa folie («La memoria crudel de'miei furori») en donnant Aspasia à Sifare - dans un simple récitatif assez intéressant. Repentant, désormais loyal à son père, Farnace n'a plus qu'à se jeter aux pieds de Mitridate, qui lui rend sa tendresse et meurt heureux, complètement heureux, chante-t-il: «Basta cosi: moro felice appieno» (III, 12). On imagine mal une telle déclaration dans la bouche du redoutable héros de Racine!

Sifare a donc son Aspasia, Farnace son Ismene: tous les personnages sont revenus au bien et tous, sauf Mitridate, goûtent le bonheur possible. Aspasia avait raison de chanter dans sa cavatine 21 (III, 4) que l'innocence bénéficie toujours de la faveur des dieux («No, l'innocenza i Numi ha sempre in suo favor»). Quelle tragédie reste-t-il dans cette réconciliation générale au sein de la vertu?

De manière encore plus éclatante, Idomeneo récuse par son dénouement une donnée tragique pourtant magnifiquement rendue sensible par Mozart. Il faut se souvenir de l'horreur des dénouements dans les tragédies françaises qui dramatisaient le mythe: chez Crébillon ${ }^{13}$, Idamante se tue $(\mathrm{V}, 5)$ et chez Danchet, source de Varesco, 
Idoménée, rendu fou par les dieux, immole lui-même son fils (V, 5); les dieux obtiennent leur victime innocente.

Il en va tout autrement chez Mozart, où deux surprises marquent le dénouement ${ }^{14}$. Au moment où Idomeneo va sacrifier son fils, Ilia survient, arrête le bras d'Idomeneo et s'offre en victime à la place de son aimé. Cet acte provoque la seule intervention chantée du divin dans l'opéra. Dans un fracas souterrain, la statue de Neptune s'anime, le Grand Prêtre entre en transe et la Voce souterraine («una voce profonda e grave»), effrayante, encadrée et ponctuée seulement par trois trombones et deux cors placés à l'endroit même d'où part la voix, chante ou psalmodie la sentence divine. Le sens est le suivant: le geste d'Ilia détermine le Ciel à abandonner son exigence et à pardonner à Idomeneo, qui cependant devra abdiquer au profit de son fils. De cette abdication et du mariage entre Ilia et Idamante Neptune sera satisfait. Ainsi, l'innocence est épargnée, récompensée, et la paix promise au royaume de Crète. L'opéra peut donc s'achever dans la joie.

Dénouement parfaitement assumé par Mozart et en germe depuis le début de l'opéra; et il faut y voir le refus du tragique, sous sa forme la plus grecque qui soit, les dieux n'ayant pas pour habitude de revenir sur leurs décrets et la loi implacable et injuste du destin menant inexorablement à la mort. Mozart refuse cette tragédie-là. «Ha vinto l'amore», chante la Voce; les dieux sont vaincus par l'amour des innocents, par l'amour offert d'Ilia.

Et ils pardonnent, dénouent le vœu d'Idomeneo. Dès le début de l'opéra s'exercent la compassion, la clémence, toute idée de vengeance est abandonnée, aucune passion dévastatrice - sauf chez la tragique Elettra - ne subsiste. Tout tend à la victoire de l'amour et de la clémence, chez les hommes et chez les dieux. Il fallait que s'instaurent l'apaisement, la réconciliation et le bonheur. Il faut encore croire à des amours heureuses.

Quand on considère les tragédies ou les livrets français qui ont traité la même fable d'Idoménée, la position originale de Mozart se détache avec netteté.

Crébillon (1705), toujours à la recherche de l'horreur et du pathétique, et Danchet (1712), qui partit de la pièce de Crébillon, n'ignorent pas l'étrangeté du sombre mythe pour la mentalité de leurs contemporains. Ils en respectent néanmoins l'atroce déroulement et la signification - la soumission des hommes à la fatalité -, l'un par goût de l'horreur, l'autre par goût pour l'Antiquité. Quand, en 1764, Lemierre écrit son propre Idoménée, il dénonce en homme des Lumières le mythe qu'il reprend sans en changer notablement la trame. L'histoire d'Idoménée donne une belle illustration du «fanatisme antique», et permet à Lemierre de s'en prendre à ces dieux honteux qui se déshonorent par des sacrifices humains, chez un peuple barbare et superstitieux ${ }^{15}$.

Mozart, qui n'est pas voltairien, mais bien un homme des Lumières, en use autrement. Il prend au sérieux, d'abord, la fatalité tragique et suit ses sources françaises. Mais le Dieu de Mozart ne peut être le Neptune du mythe. Chrétien et bientôt maçon, Mozart ne peut croire à un Dieu vindicatif et sanguinaire; mais il croit au Dieu bon, généreux, qui aime les hommes et veut leur bonheur. D'où l'intervention imprévue de la Voce qui parle non de sacrifice mais d'amour, de pardon et de paix. Il bouleverse donc le dénouement reçu et sa signification, supprimant et dépassant le tragique - à la vérité contesté dès l'ouverture avec sa fragile promesse d'éclaircie, contesté tout au long de l'opéra, et définitivement vaincu dans le chœur final.

(14) Précisions dans Ch. Mazouer, "Idomeneo, rè di Creta": Mozart et la tragédie, cit., pp. 141142 .
(15) Voir aussi La Veuve de Malabar, dont la traduction allemande fut rattrapée par la musique de Thamos. 
Bien au-delà de la reprise des fables ou de topoï éculés, les changements de l'intrigue, les déplacements d'accent et d'intérêt, les bouleversements du dénouement par rapport aux tragédies françaises, et à travers eux le renouvellement de la signification donnée aux histoires reprises de nos tragiques, signalent assez une autre vision du monde. Mozart répugne finalement au tragique qu'il est capable de ressentir et qu'il donne à entendre dans sa musique; il le surmonte et le dépasse. Mais je voudrais laisser le mot de la fin au grand théologien luthérien allemand Karl Barth ${ }^{16}$, dont le propos me semble d'une très grande justesse. «Au fond, il n'a jamais été vraiment tragique», dit-il de Mozart ${ }^{17}$. Et de donner plus loin ces précisions: «Aucune clarté à laquelle l'ombre ne serait familière, nulle joie qui ne comporte sa souffrance, et inversement: pas d'effroi, pas de colère, pas de plainte qui ne soient accompagnés, de près ou de loin, par la paix! $\gg^{18}$.

CHARLES MAZOUER

(16) Wolfgang Amadeus Mozart: 1756-1956, Genève, Labor et Fides, 1969, $2^{\circ}$ éd. (1956).
(17) p. 35

(18) p. 43. 